\title{
Communicating the role of academia in the control of non- communicable diseases: identification, involvement and institutionalization
}

Vaidya $\mathrm{A}^{1}$

Two out of every three people in the world die of non-communicable diseases (NCDs), mostly from cardiovascular diseases, diabetes mellitus, chronic lung diseases, and cancer (1). Four out of five NCD-related deaths occur in the low- and middle-income countries and it is expected that this proportion will rise in the future (2). In Nepal, 60 percent of disease burden is attributed to NCDs (3), which should not be surprising for the fact that the risk factors such as tobacco and alcohol consumption, indoor air pollution, blood pressure, and body mass index are high in the Nepalese population (4), while the health literacy is low (5) and health promotional activities are sporadic and cure-centric (6).

Focused almost entirely on infections, malnutrition and reproductive health for most of the Millennium Development Goals period (2000-2015), it is only recently that the global health leaders have woken up to the severity of the NCD-situation and its consequences (7). Nevertheless, foundations are now being laid on a global scale to plan and implement strategies to curb the NCDs (8), with clear targets of bringing down the impact of NCDs by 2025 (9). The global targets have been adapted down to regional levels, and then further to country-levels, Nepal included (10).

NCDs are multi-factorial in origin, and their control should therefore also be multi-sectoral. For this, in accordance with the global consensus, four key strategic action areas have been identified in the Nepal Multi-sectoral action plan for the Prevention and Control of NCDs (11): Area 1 (Advocacy, partnership and leadership); Area 2 (Healthy lifestyle and reduction of major NCD risk factors); Area 3 (Health system strengthening for early detection and management of NCDs); and Area 4 (Surveillance, research, monitoring and evaluation).

Three groups of stakeholders have their roles to play in these strategic action areas: The State (Ministry of Health and Population; and other ministries such as Ministry of Education, Ministry of Youth, Ministry of Transport, etc); the Internal support system (Non-governmental organizations, civil society organizations, academia and private sectors); and the External support system (External development partners, World Bank, funding agencies, etc). While it is the State that formulates policies and plans, and implements them through the ministries, the internal and external support systems provide necessary assistance to the State at different levels. For example, World Health Organization, an External development partner, supported the Ministry of Health and Population of Nepal in making the Multi-sectoral Action Plan for the NCDs. Similarly, Nepal Public Health Foundation, a civil society organization, was instrumental in enhancing stakeholders' attention to NCDs by organizing regional and national seminars on NCDs (12).

In this regard, the role of academics in NCD control is also clearly chalked out in the Nepal Multi-sectoral action plan for the Prevention and Control of NCDs, in the sense that the academic institutions are to be involved mainly in research activities that include building its research capacity, doing secondary data analysis on the existing data sources and thus providing evidence for program and policy (11). However, the Nepalese academic community is quite sporadic and heterogeneous in terms of its interest and exposure to NCD-related researches. It is more of the individuals within the institutions, rather than the institutions themselves who have been involved in the NCD-related research or policy-drafting. So, it is important that the individual-level researchers and their research works be institutionalized. Furthermore, these individual NCD focal-points must be joined together by a line that brings them together, so that the 'NCDacademia' of Nepal becomes more recognizable and perceptible. In lack of a mechanism that can collate and compile the past and ongoing research works from the academic institutions, the researchers and their research works may not come into the radar of

\author{
Correspondence: \\ ${ }^{1}$ Abhinav Vaidya (MD, PhD) \\ Associate Professor (Community Medicine) \\ Department of Community Medicine \\ Kathmandu Medical College \\ Kathmandu, Nepal. \\ P.O.Box: 21266
}

Email: dr.abhinavaidya@gmail.com 
the core stakeholders such as the Ministry of Health and Population, Nepal.

Though the primary strength of the academia lies in its input to the strategic area 4 (Surveillance, research, monitoring and evaluation), it should not limit itself only to this area. Academia can contribute to the Area 1 (Advocacy, partnership and leadership) by, for example, partnering with other members of the internal support system for advocacy of NCD-related issues. Training of health-manpower on NCD-related activities is another potential collaboration. Similarly, the academia can support Area 2 (Healthy lifestyle and reduction of major NCD risk factors) by two ways: by incorporating health promotional activities in their academic endeavours including community-based programmes; and by undertaking research-works that intend to reduce NCD risk factors at community level. Likewise, through its skilled manpower, academic institutions with clinical settings can help contribute to Area 3 (Health system strengthening for early detection and management of NCDs).

Having said these, the academia must also be well prepared to undertake the possible greater responsibility in NCD-related works, especially when there is inevitable increase in attention and funding from the global and regional agencies (13). While all these roles of academia have been well-stressed at the global platform in terms of seeking responsibilities, recommendations and responses (14), it is critical that the same be pursued at the local level as well.

\section{References}

1. Lozano R, Naghavi M, Foreman K, AIMazroa MA, Memish ZA. Global and regional mortality from 235 causes of death for 20 age groups in 1990 and 2010: a systematic analysis for the Global Burden of Disease Study 2010. Lancet. 2012;380:2095-2128.

2. Alwan A. Global status report on noncommunicable diseases 2010. Geneva: World Health Organization; 2011.

3.World Health Organiation. Disease and country estimates. Geneva: World Health Organiation; 2013. Available at: http://www.who.int/healthinfo/ global_burden_disease/estimates_country/en/index.html.

4. Aryal KK, Neupane S, Mehata S, Vaidya A, Singh S, Paulin F, et al. Nepal non-communicable diseases risk factors: STEPS survey Nepal 2013. Kathmandu: Nepal Health Research Council.

5. Vaidya A, Aryal UR, Krettek A. Cardiovascular health knowledge, attitude and practice/behaviour in an urbanising community of Nepal: a population-based cross-sectional study from Jhaukhel-Duwakot Health Demographic Surveillance Site. BMJ open. 2013;3(10):e002976.

6. Vaidya A, Krettek A. Is Health Promotion the Starting Point of Primary Cardiovascular Care in Low-and Middle-Income Countries Like Nepal? Health Promotion Practice. 2012;13(3):412-5.

7. Beaglehole R, Bonita R, Alleyne G, Horton R, Li L, Lincoln P, et al. for The Lancet NCD Action Group. UN high-level meeting on non-communicable diseases: addressing four questions. Lancet. 2011;378:449-55.

8. World Health Organiation. 2008-2013 action plan for the global strategy for the prevention and control of noncommunicable diseases. Geneva: World Health Organization; 2008.

9. World Health Organiation. Global action plan for the prevention and control of noncommunicable diseases 2013-2020. Geneva: World Health Organiation; 2013.

10. Ministry of Health and Population, Nepal. Integrated Non-Communicable Diseases (NCDs) Prevention and Control Policy of Nepal. Kathmandu: Ministry of Health and Population; 2010.

11. Ministry of Health and Population, Nepal. Multisectoral Action Plan for the Prevention and Control of Non Communicable Diseases (2014-2020). Available from: http://www.searo.who.int/nepal/mediacentre/ncd_multisectoral_action_plan.pdf.

12. Nepal Public Health Foundation. National workshop on Non-communicable Diseases. Kathmandu: Nepal Public Health Foundation; 2011.

13. Greenberg H. Global Cardiovascular Disease and the Academic Public Health Curriculum. Progress in cardiovascular diseases. 2013;56(3):322325.

14. United Nations. Informal interactive hearing with non-governmental organizations, civil society organizations, the private sector and academia on the prevention and control of noncommunicable diseases (NCDs). Available from: http://www.idf.org/sites/default/files/692014Draft-Concept_ note-Informal_interactive_hearing.pdf. 